\title{
Surface CD200 and CD200R antigens on lymphocytes in advanced gastric cancer: a new potential target for immunotherapy
}

\author{
Witold Zgodziński ${ }^{1}$, Ewelina Grywalska², Agata Surdacka², Krzysztof Zinkiewicz ${ }^{1}$, \\ Marek Majewski ${ }^{1}$, Dariusz Szczepanek ${ }^{3}$, Grzegorz Wallner ${ }^{1}$, Jacek Roliński
}

\begin{abstract}
${ }^{1} 2^{\text {nd }}$ Department of General, Gastrointestinal Surgery and Surgical Oncology of the Alimentary Tract, Medical University of Lublin, Lublin, Poland ${ }^{2}$ Department of Clinical Immunology and Immunotherapy, Medical University of Lublin, Lublin, Poland

${ }^{3}$ Department of Neurosurgery and Pediatric Neurosurgery, Medical University of Lublin, Lublin, Poland
\end{abstract}

Submitted: 28 February 2017

Accepted: 1 September 2017

Arch Med Sci 2018; 14, 6: 1271-1280

DOI: https://doi.org/10.5114/aoms.2018.73398

Copyright @ 2018 Termedia \& Banach

\section{Abstract}

Introduction: Gastric cancer (GC) is one of the leading causes of cancer death worldwide. The membrane glycoprotein CD200, widely expressed on multiple cells/tissues, uses a structurally similar receptor (CD200R), delivering immunoregulatory signals. There is evidence that CD200/CD200R signaling suppresses anti-tumor responses in different types of malignancies. Little is known about the CD200/CD200R pathway in GC. The aim of the study was to evaluate the frequencies of CD200+ and CD200R+ lymphocytes in patients with GC.

Material and methods: Forty patients primarily diagnosed with GC and 20 healthy volunteers (control group) were enrolled. The viable peripheral blood lymphocytes underwent labeling with fluorochrome-conjugated monoclonal antibodies and were analyzed using a flow cytometer.

Results: In the GC group, the percentages of T CD3+, CD3+/CD4+, and CD3+/ CD8+ cells expressing CD200 antigen were higher than in the control group ( $p<0.00013, p<0.0004$, and $p<0.0006$, respectively). In the GC group, the frequencies of $\mathrm{T} \mathrm{CD} 3+, \mathrm{CD} 3+/ \mathrm{CD} 4+$ and CD3+/CD $8+$ cells expressing CD200R were lower than in the control group $(p<0.0009, p<0.004$, and $p<0.002$, respectively). The percentage of B CD19+/CD200+ lymphocytes was higher in GC patients than in the control group $(p<0.00005)$. Lower frequency of B CD19+/CD200R+ cells was observed in GC patients compared to the control group $(p<0.0001)$. No differences in the frequencies of CD200+ and CD200R+ lymphocytes were found in relation to either UICC stage or histological grading of the tumors.

Conclusions: For GC pathogenesis, deregulation of the CD200/CD200R axis is important. High percentages of lymphocytes with CD200 expression may contribute to the continuous $T$ cell activation and development of chronic inflammation and influence gastric carcinogenesis.

Key words: gastric cancer, CD200, lymphocytes, CD200R molecules.

\section{Introduction}

The fourth most prevalent cancer worldwide is gastric cancer (GC), and it is the second most common cause of cancer deaths [1]. In the past decades, much progress has been made in therapeutic procedures,

\author{
Corresponding author: \\ Witold Zgodziński MD, PhD \\ $2^{\text {nd }}$ Department \\ of General, \\ Gastrointestinal Surgery \\ and Surgical Oncology \\ of the Alimentary Tract \\ Medical University \\ of Lublin \\ 16 Staszica St \\ 20-081 Lublin, Poland \\ Phone: +48 815324127 \\ +48 815324127 \\ Fax: +48 815328810 \\ E-mail:wzgodz@wp.pl
}


including surgery, chemotherapy, and radiotherapy, but the treatment outcomes are still very poor, with relatively high mortality compared to other gastrointestinal malignancies. Ineffective screening, late diagnosis, and primary and/or acquired resistance to chemotherapy/radiotherapy are the most important causes of treatment failure for GC. This disease is complex in its biology because it arises from different interactions of genetic, immunological, environmental, and host factors, resulting in high heterogeneity. Therefore, in order to improve the treatment results of GC, understanding the biological characteristics at different levels is crucial.

At present, the concept of tumor-induced immune deregulation of the host - as one of the mechanisms involved in cancer progression - is being widely discussed. Various experimental and clinical data confirm that immunocompromised patients are at higher risk of developing cancers [2-4]. On the other hand, there is a positive correlation between the presence of lymphocytes in tumor tissue and increased patient survival [5]. In order to evade immunosurveillance, tumors develop special mechanisms including the loss of tumor antigen expression, the expression of Fas ligand (Fas-L) or CD200 [6]. CD200 is a type 1a membrane protein with two extracellular Ig superfamily domains, a single transmembrane region, and a short cytoplasmic tail [7]. It is broadly expressed on a variety of cell types, including T and B lymphocytes [8]. CD200 delivers immunoregulatory signals by binding to receptors expressed on monocytes/myeloid cells, including monocyte-derived dendritic cells, and T lymphocytes. Signals delivered via the CD200/CD200R axis were shown to play an important role in regulation of the anti-tumor immune response. In a number of malignancies, overexpression of CD200 was found [9-11], and it was suggested that it may play a role in invasion and metastases of certain types of cancer [12]. In gastric cancer, the possible role of CD200 was not studied. The aim of the present study was to assess the expression of CD200 and CD200R on lymphocyte subsets in peripheral blood in patients suffering from gastric cancer compared to healthy donors. To the best of our knowledge, the present publication is the first to describe the mentioned issue. The results of our study may help to better understand the immune deregulation in GC patients, particularly in the aspect of lymphocyte subsets, and to contribute to setting new standards for cancer immunotherapy in the future.

\section{Material and methods}

\section{Patients and healthy controls}

Forty consecutive patients (33 male and $7 \mathrm{fe}$ male) treated for primarily diagnosed advanced gastric adenocarcinoma, without any previous, neoadjuvant treatment, were included in the study. The diagnosis of GC was established by histopathology of tumor endoscopic biopsies. The staging was performed postoperatively using Union International for Cancer Control (UICC) TNM staging ( $7^{\text {th }}$ Edition) [13]. The mean age of patients was $61.85 \pm 10.07$ years (ranging from 40 to 77 years; median: 63). Peripheral blood (PB) from 20 healthy donors, at the mean age of 56.83 \pm 10.24 (ranging from 43 to 70 years; median: 57 ), was used as a control. Both in patients and healthy donors, peripheral blood white blood cell count was within the normal range between 4 and $10 \mathrm{G} /$ l. None of the patients or controls showed signs of infection at the time of investigation, and for a month before surgery, none had been taking drugs of known influence on the immune system. None of the patients or healthy donors had undergone blood transfusion. Individuals with allergic diseases in the anamnesis and other current or past oncological/oncohematological disease in the anamnesis were excluded from the study. The research protocol was approved by the local Ethics Committee (decision no. KE-0252/176/2013), and all patients gave written informed consent.

\section{Isolation of peripheral blood cells and the detection of CD200-positive and CD200R-positive T and B lymphocytes}

Approximately 5-ml venous blood samples were collected from the study patients (1 day before surgery) and controls by venipuncture using sterile, lithium heparin-treated S-Monovette tubes (Sarstedt, Nümbrecht, Germany). One hour after obtaining blood samples from the patients and controls, PB mononuclear cells were aseptically separated by standard density gradient centrifugation (Gradisol L, Aqua Med, Lodz, Poland). The percentages of cells expressing surface markers were analyzed. The cells were phenotypically characterized by incubation ( $20 \mathrm{~min}$ in the dark at room temperature) with a combination of relevant fluorescein isothiocyanate (FITC) - phycoerythrin (PE) - and CyChrome-labeled monoclonal antibodies (mAbs). Using a combination of the following mAbs, immunofluorescence studies were performed: CD45 FITC/CD14 PE, CyChrome Mouse Anti-Human CD3, FITC Mouse Anti-Human CD19, FITC Mouse Anti-Human CD4, FITC Mouse AntiHuman CD8, PE Mouse Anti-Human CD200, and PE Mouse Anti-Human CD200R, purchased from BD Biosciences (San Jose, CA, USA). Using a FACSCalibur flow cytometer (Becton Dickinson, San Diego, CA, USA) equipped with 488-nm argon laser, three-color immunofluorescence analyses were performed. Using CellQuest Software, a minimum of 10,000 events were acquired and analyzed. 
The results were presented as the percentage of CD45+ cells stained with antibody. The percentage of positive cells was calculated by comparing with the control. Using isotype-matched directly conjugated FITC Mouse IgG1 $\kappa$ Isotype Control and PE Mouse IgG1 $\kappa$ Isotype Control monoclonal antibodies, background fluorescence was determined. In order to exclude debris and cell aggregates, the samples were gated on forward scatter vs. side scatter. Figure 1 presents an example of the cytometric analysis.
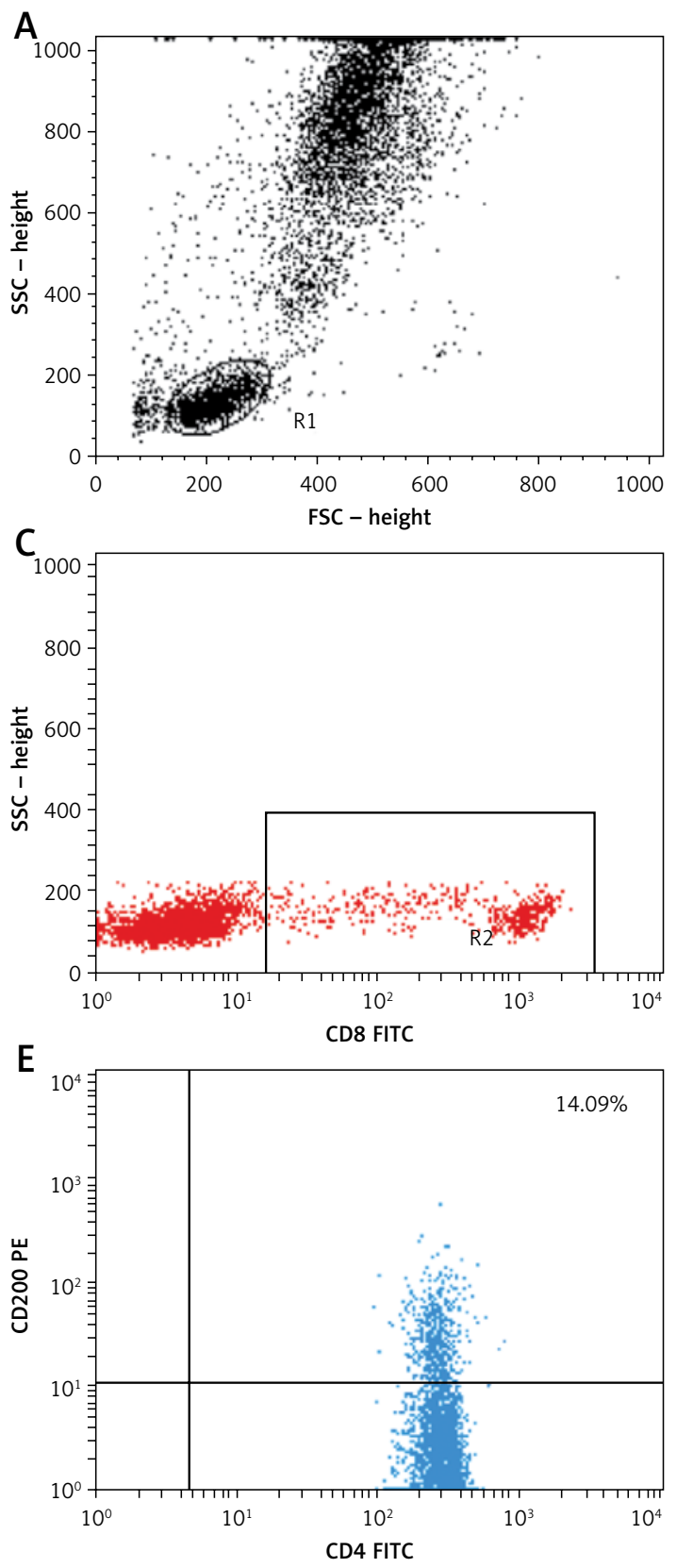

\section{Statistical analysis}

The normal distribution of continuous variables was verified with the Shapiro-Wilk test. Frequencies of the cells were presented as percentages, and continuous variables were presented as means and standard deviations. The Mann-Whitney $U$ test and Student's $t$-test were applied to intergroup comparisons of the variables, according to the distribution. Pearson's linear correlation coefficient $(r)$ was calculated to assess relationships between variables. All calculations were
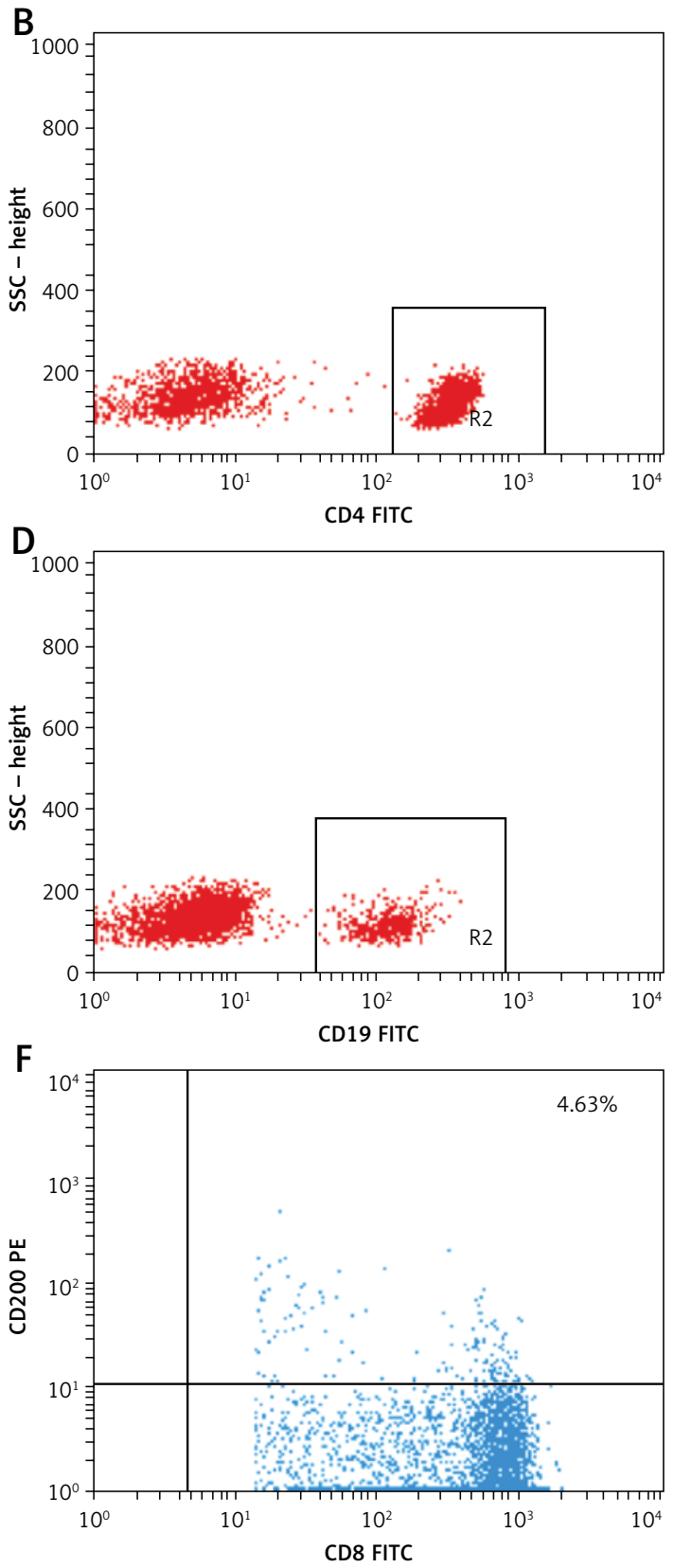

Figure 1. Example of a three-color flow cytometry analysis of CD200 antigen expression on lymphocytes from a patient with gastric cancer. The upper dot plot shows the forward scatter/side scatter (FSC/SSC) distribution and the gate used to select lymphocytes for analysis. The middle row dot-plots represent an example of a healthy control. The lower row dot-plots represent an example of a GC patient. The numbers in the upper right quadrant in the dot-plots show the percentage of CD200+ cells 

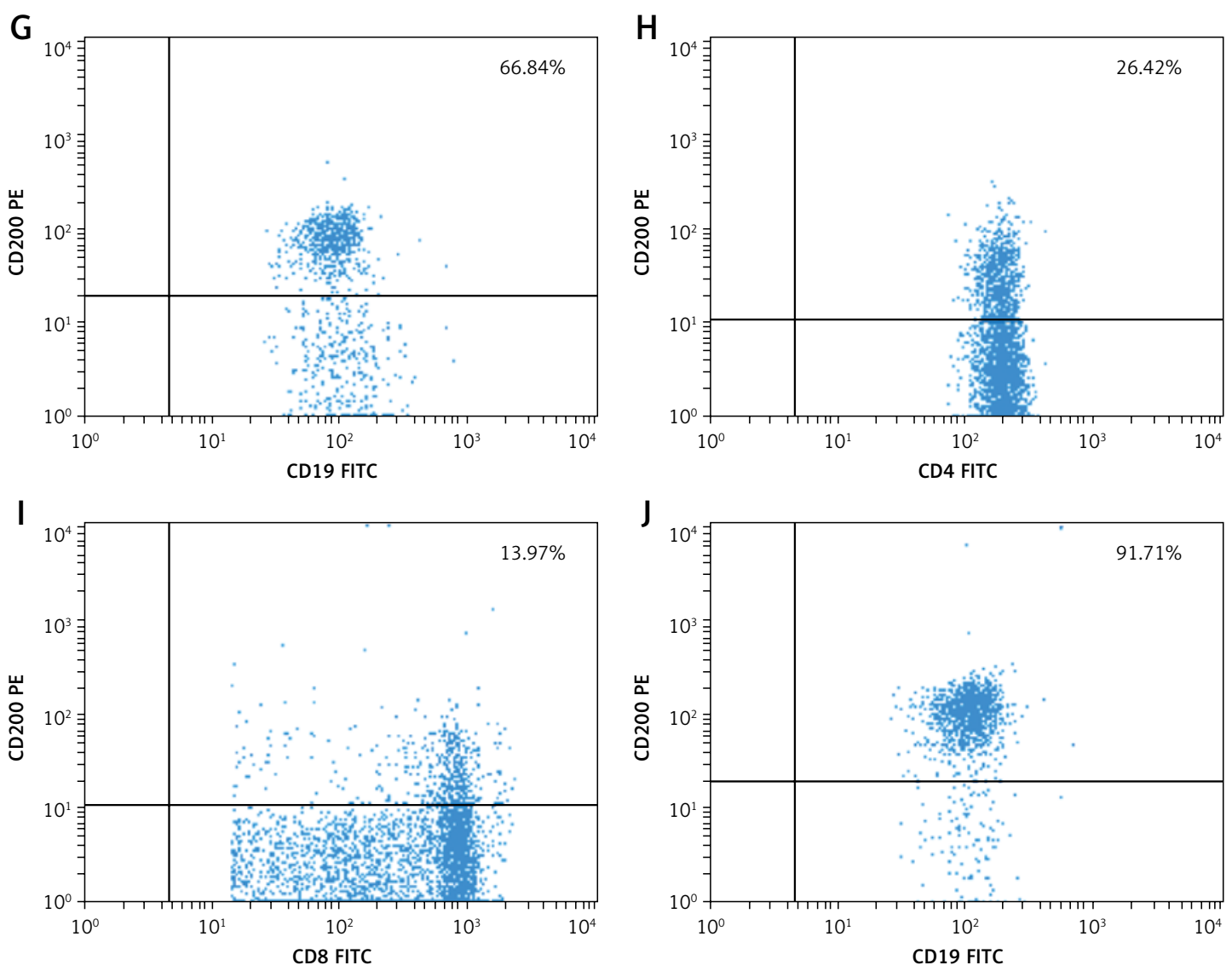

Figure 1. Cont.

Table I. Clinical and pathological characteristic of the studied gastric cancer patient group $(n=40)$

\begin{tabular}{|c|c|c|c|c|}
\hline \multicolumn{3}{|c|}{ Parameter } & \multicolumn{2}{|c|}{ Value } \\
\hline \multicolumn{3}{|c|}{ Age, mean \pm SD [years] } & \multicolumn{2}{|c|}{$61.85 \pm 10.07$} \\
\hline & & & $N$ & Percentage \\
\hline \multirow{2}{*}{\multicolumn{2}{|c|}{ Sex }} & Male & 33 & 82.5 \\
\hline & & Female & 7 & 17.5 \\
\hline \multirow{7}{*}{$\begin{array}{l}\text { Staging } \\
\text { (UICC } 7^{\text {th }} \\
\text { Edition) }\end{array}$} & I & B & 1 & 2.5 \\
\hline & II & A & 7 & 17.5 \\
\hline & & B & 6 & 15 \\
\hline & III & A & 11 & 27.5 \\
\hline & & B & 3 & 7.5 \\
\hline & & C & 5 & 12.5 \\
\hline & IV & & 7 & 17.5 \\
\hline \multirow{3}{*}{\multicolumn{2}{|c|}{$\begin{array}{l}\text { Grading } \\
\text { (histological } \\
\text { differentiation } \\
\text { of tumor) }\end{array}$}} & G1 & 6 & 15 \\
\hline & & G2 & 10 & 25 \\
\hline & & G3 & 24 & 60 \\
\hline \multirow{2}{*}{\multicolumn{2}{|c|}{$\begin{array}{l}\text { Lauren } \\
\text { classification }\end{array}$}} & Intestinal & 22 & 55 \\
\hline & & Diffuse & 18 & 45 \\
\hline
\end{tabular}

conducted using Statistica 10 software (StatSoft, USA). A $p$-value less than 0.05 was considered statistically significant.

\section{Results}

Table I presents patients' characteristics. The results are presented in the form of percentage values of the cells presenting the expression of a given antigen and the mean fluorescence intensity (MFI), which is the mean score of the density of expression of a given molecule on a cell. Our study revealed that in the GC group, the percentage of T lymphocyte subsets with CD200 expression including CD3+ (median: 23.98\%; range: 12.447.21\%), CD4+ (median: $13.56 \%$; range: $3.47-$ $37.1 \%$ ) and CD8+ (median: 9.84\%; range: $2.33-$ $29.16 \%$ ) was significantly higher than in the respective control group, that is, CD3+ (median: 8.87\%; range: $1.55-14.57 \% ; p<0.00013$ ) (Figure $2 \mathrm{~A}$ ), CD4+ (median: 4.86\%; range: 1.05-8.95\%; $p<0.0004$ ) (Figure $3 \mathrm{~A}$ ), and CD8+ (median: $2.76 \%$; range: $0.35-5.02 \% ; p<0.0006$ ) (Figure $4 \mathrm{~A}$ ). In the $\mathrm{GC}$ group, the percentage of $\mathrm{T}$ lymphocyte subsets with CD200R expression including CD3+ (median: $19.49 \%$; range: $5.62-40.71 \%)$, CD4+ (median: 11.59\%; range: $3.23-23.82 \%$ ), and CD8+ (median: $4.98 \%$; range: $0.59-13.89 \%)$ was significantly 
A

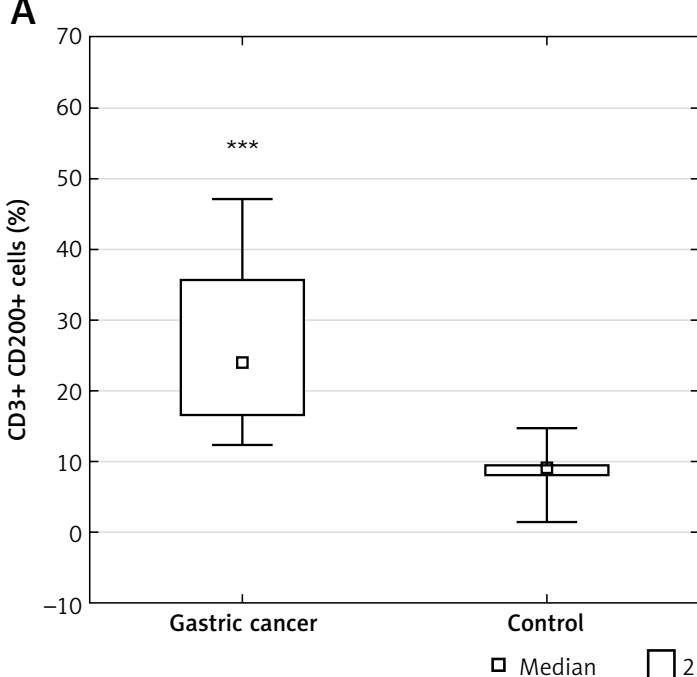

B

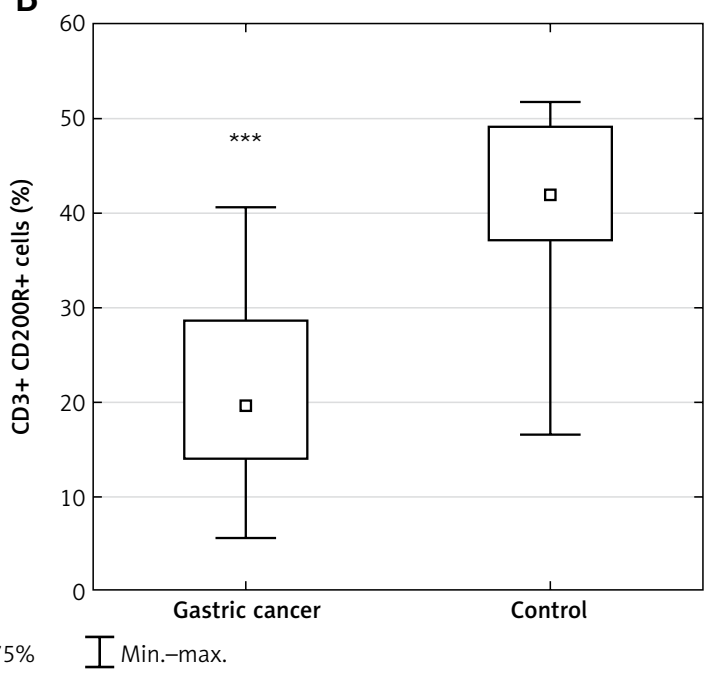

Figure 2. Diagrams presenting: A - the difference between the percentage of CD3+/CD200+ lymphocytes in gastric cancer (GC) patients and healthy controls ( ${ }^{* * *} p<0.00013 \mathrm{vs}$. control); $\mathrm{B}$ - the difference between the percentage of CD3+/CD200R+ lymphocytes in GC patients and healthy controls ( ${ }^{* * *} p<0.0009$ vs. control)

A

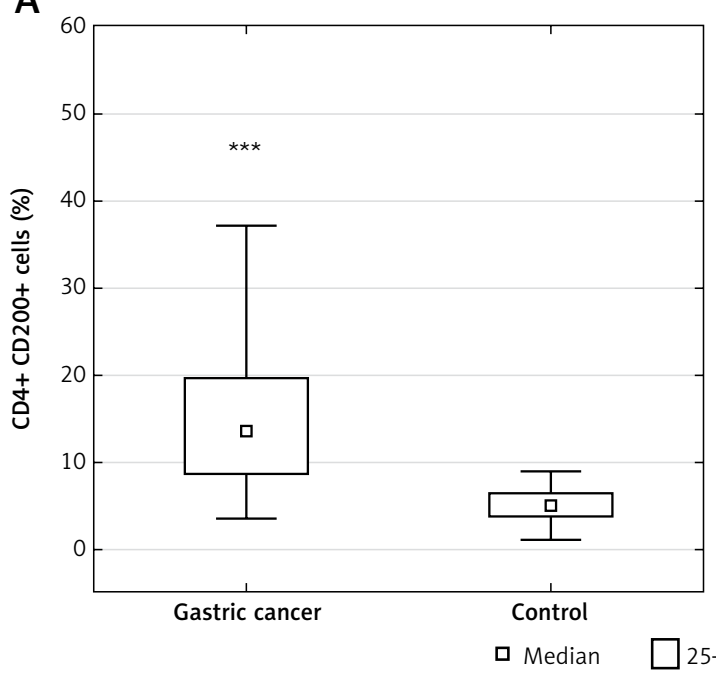

B

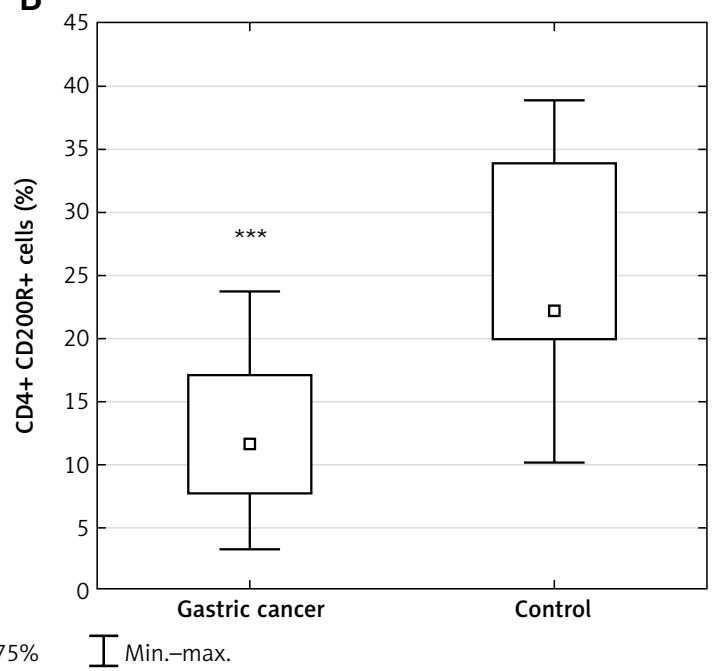

Figure 3. Diagrams presenting: A - the difference between the percentage of CD4+/CD200+ lymphocytes in gastric cancer (GC) patients and healthy controls ${ }^{* \star *} p<0.0004$ vs. control); $B$ - the difference between the percentage of CD4+/CD200R+ lymphocytes in GC patients and healthy controls ${ }^{* * *} p<0.004$ vs. control)

lower than in the respective control group, that is, $\mathrm{CD} 3+$ (median: $41.97 \%$; range: $16.47-51.7 \%$; $p<0.0009$ ) (Figure $2 \mathrm{~B}$ ), CD4+ (median: 22.17\%; range: $10.03-38.83 \% ; p<0.004$ ) (Figure $3 \mathrm{~B}$ ), and CD8+ (median: $13.12 \%$; range: $6.03-21.73 \%$; $p<0.002$ ) (Figure $4 \mathrm{~B}$ ).

In the GC group, a higher percentage of CD19+ cells with CD200 antigen expression was also observed compared to the control group (median: 99.32\%; range: $80.48-99.99 \%$ vs. median: $70.97 \%$; range: $62.51-78.79 \% ; p<0.00005)$ (Figure $5 \mathrm{~A}$ ). In the GC group, a significantly lower percentage of CD19+ cells with CD200R antigen expression was also observed compared to the control group (median: $11.09 \%$; range: $5.25-19.19 \%$ vs. median: $22.44 \%$; range: $16.91-30.66 \% ; p<0.0001$ ) (Figure $5 \mathrm{~B}$ ).
There was no difference in MFI values of CD200 antigen expression on $\mathrm{CD} 3+, \mathrm{CD} 4+$, and $\mathrm{CD} 8+$ cells between GC and control groups. MFI values of CD200 antigen on CD19+ cells in the GC group (mean: $170.69 \pm 58.64$ ) were higher compared to the control group (mean: $47.17 \pm 13.24 ; p<0.001$ ) (Figure $6 \mathrm{~A}$ ).

There was no difference in MFI values of CD200R expression on CD3+, CD4+, and CD8+ cells between GC and control groups. MFI values of CD200R expression on the CD19+ cells in the GC group (mean: $134.53 \pm 53.98)$ were higher compared to the control group (mean: $29.62 \pm 15$; $p<0.004$ ) (Figure 6 B). Subset analysis revealed higher MFI values of CD200R expression on CD8+ cells in diffuse-type tumors (mean: $40.45 \pm 10.3$ ) 
A

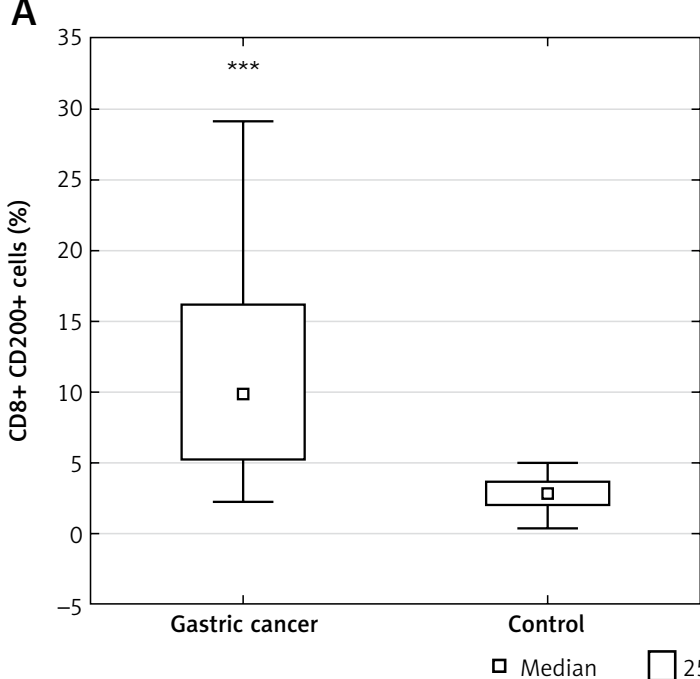

B

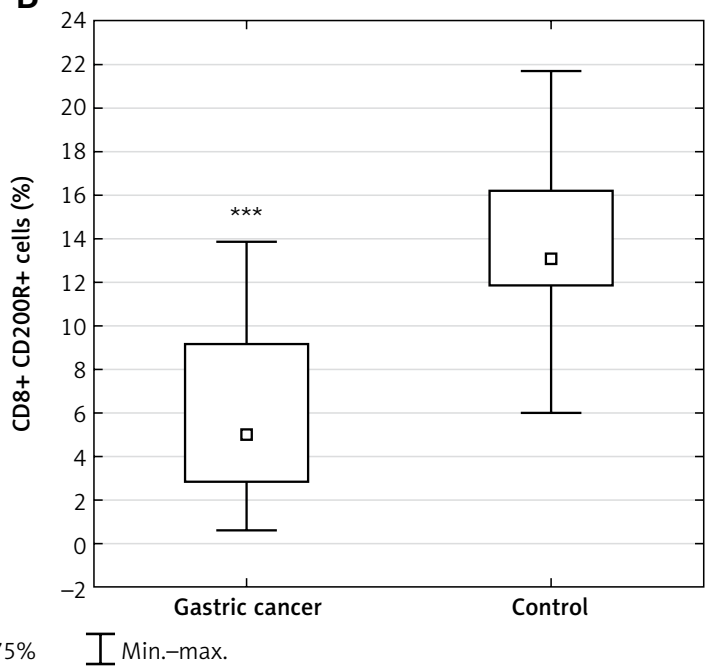

Figure 4. Diagrams presenting: $\mathrm{A}$ - the difference between the percentage of CD8+/CD200+ lymphocytes in gastric cancer (GC) patients and healthy controls ( ${ }^{* * *} p<0.0006$ vs. control); $\mathbf{B}-$ the difference between the percentage of CD8+/CD200R+ lymphocytes in GC patients and healthy controls ( ${ }^{* *} p<0.002$ vs. control)

A

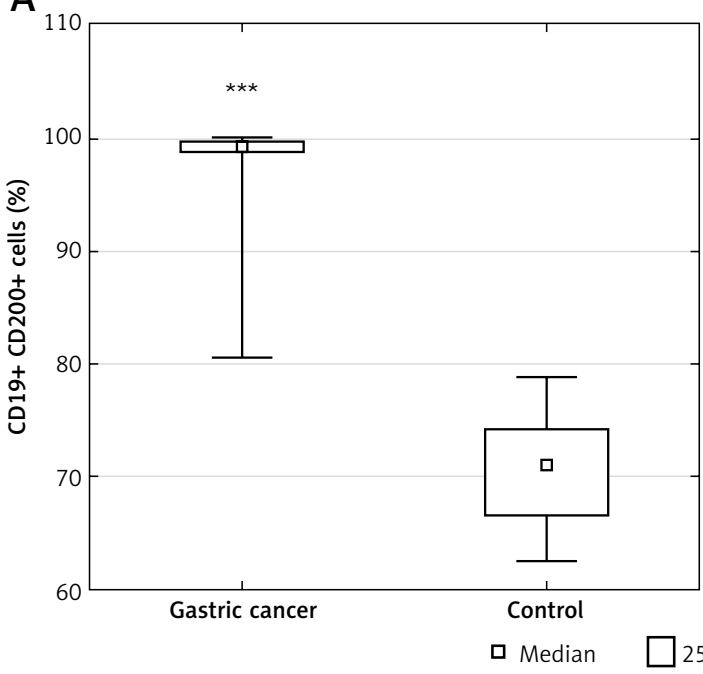

B

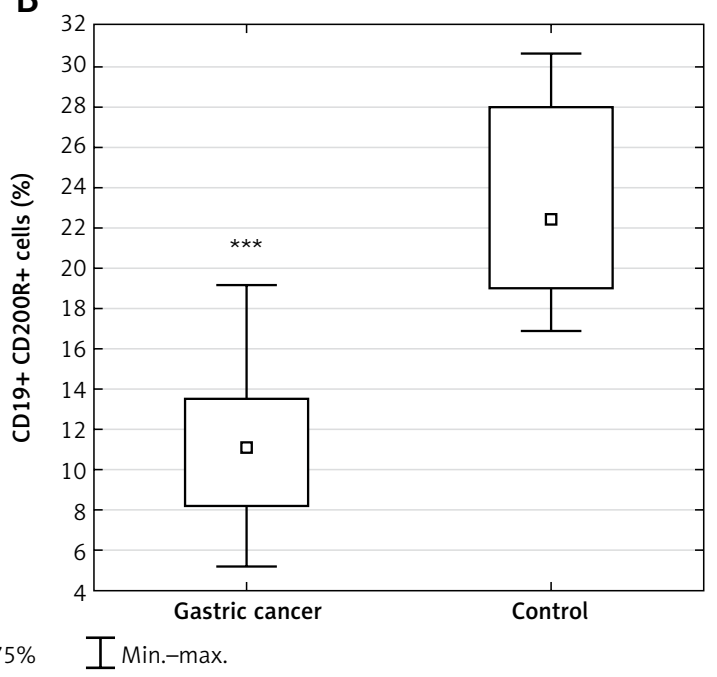

Figure 5. Diagrams presenting: A - the difference between the percentage of CD19+/CD200+ lymphocytes in gastric cancer (GC) patients and healthy controls ( ${ }^{* *} p<0.00005$ vs. control); $\mathbf{B}-$ the difference between the percentage of CD19+/CD200R+ lymphocytes in GC patients and healthy controls ( ${ }^{\star * *} p<0.0001$ vs. control)

compared to intestinal-type tumors (mean: 35.16 $\pm 28 ; p<0.05$ ).

A significant correlation in the GC group was revealed between the percentage of $\mathrm{CD} 4+$ and CD8+ cells presenting CD200 molecule expression $(r=0.707)$ (Figure $7 \mathrm{~A})$. In the GC group, higher $\mathrm{MFI}$ values of CD200 expression on CD8+ cells correlated with those on CD3+CD200+ $(r=0.606)$ (Figure 7 B). The MFI values of CD200+ on CD19+ cells correlated with MFI values of CD200R+ on CD19+ cells in the GC group ( $r=0.502)$ (Figure 7 C).

No significant differences in percentage of CD200+ and CD200R+ cells were found between patients at UICC stage I/II and III/IV. The percentage of CD200+ and CD200R+ cells in patients with well and moderately differentiated tumors (G1/ G2) was not different than in patients with $G 3$ tumors. The percentage of CD200+ and CD200R+ cells in patients with intestinal-type tumors (according to the Lauren classification) was not different than in patients with diffuse-type tumors.

\section{Discussion}

The aim of the present study was to assess the expression of CD200 and CD200R on lymphocyte subsets in PB in patients suffering from advanced gastric cancer.

Our results showed that the percentage of $T$ lymphocytes such as CD3+, CD4+, and CD8+ expressing the CD200 molecule in patients with 
A

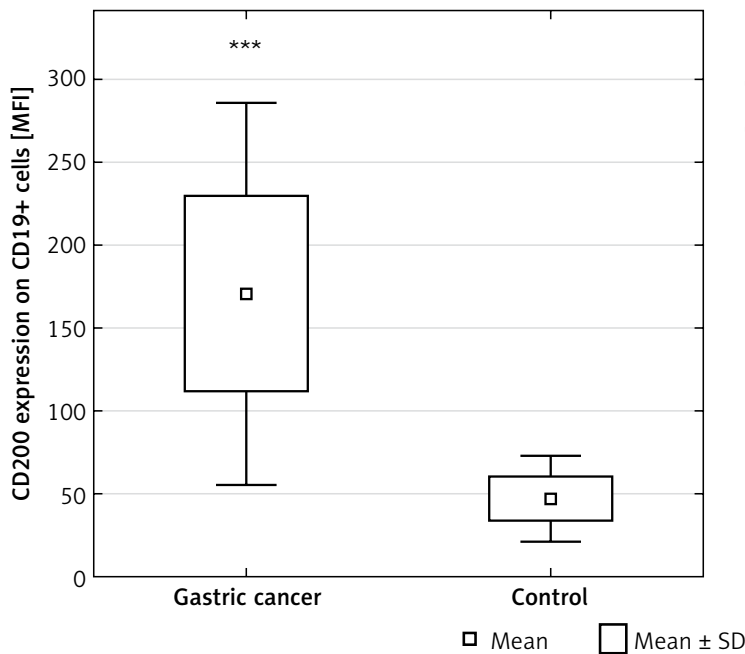

B

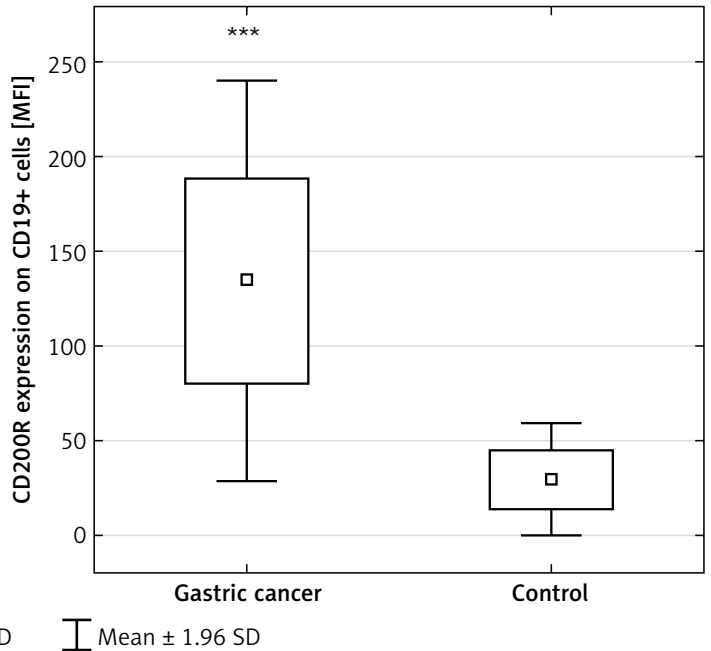

Figure 6. Diagrams presenting: $A$ - the difference between the expression of the CD200 molecule (MFI) on CD19+ cells in gastric cancer patients and healthy controls ( ${ }^{* *} p<0.001$ vs. control); $\mathbf{B}-$ the expression of the CD200R molecule (MFI) on CD19+ cells in gastric cancer patients and healthy controls ( ${ }^{\star \star *} p<0.004$ vs. control)

A

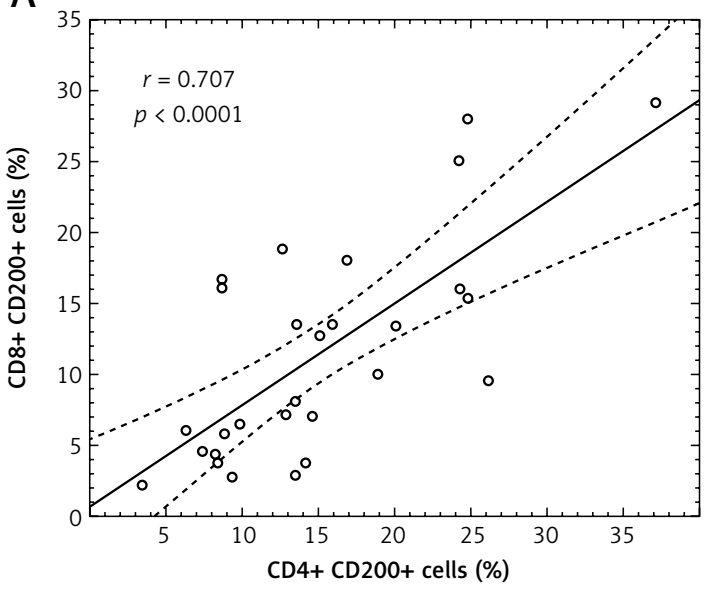

C

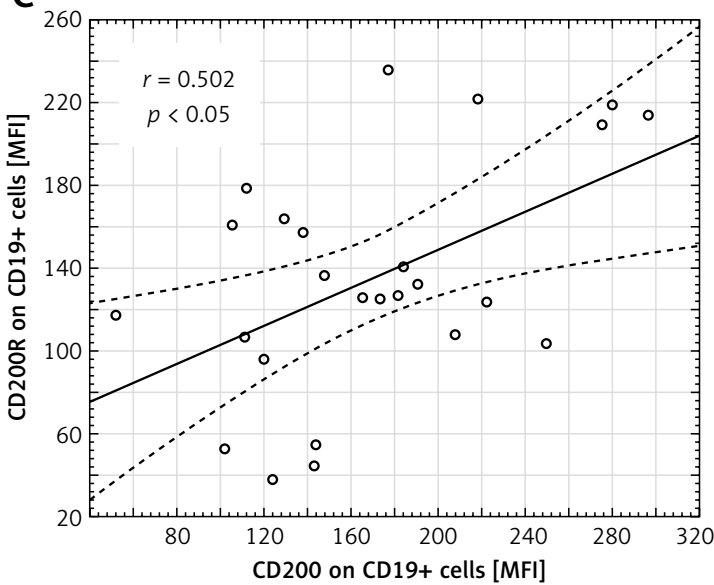

B

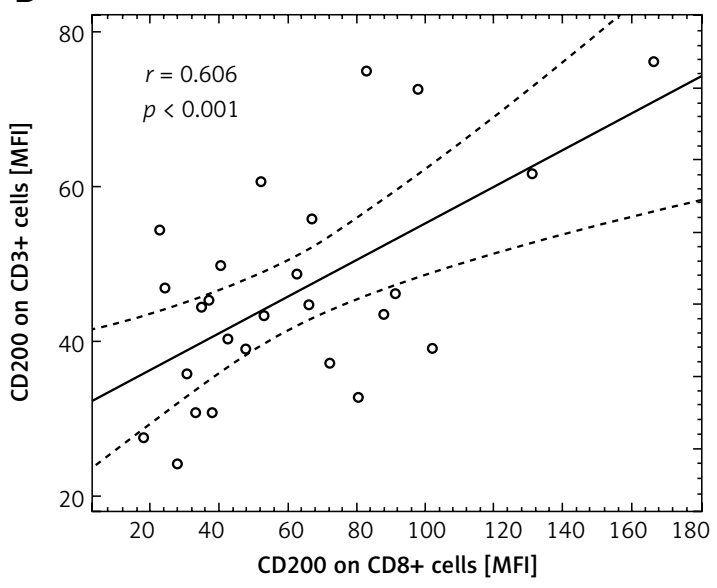

Figure 7. Scatter diagrams presenting positive correlations found in GC patients between: the percentage of CD4+ and CD8+ cells presenting CD200 molecule expression (A); MFI values of CD200 expression on $\mathrm{CD} 8+$ and $\mathrm{CD} 3+$ cells (B); MFI values of CD200 and CD200R expression on CD19+ cells (C)

advanced gastric cancer is significantly higher compared to healthy controls. Conversely, the percentage of $\mathrm{CD} 3+, \mathrm{CD} 4+$, and CD8+ cells expressing the CD200R+ molecule was lower than in

the control group. A positive correlation between CD4+ and CD8+ cells presenting the CD200 molecule is another notable result. These findings confirm the possible regulatory effect of the CD200/ 
CD200R axis on the T lymphocyte-dependent immune response in GC. The high percentage of CD200-presenting CD3+ cells - including subsets of CD4+ and CD8+ cells with a lower percentage of respective CD200R+ cells - may possibly confirm inhibition of the CD3+-dependent immune response in GC patients. Previous studies indicated a significant increase in percentage of CD3+ cells in GC compared to the control group, with a higher $C D 4+/ C D 8+$ ratio that could partly explain anti-tumor immunity against GC [14]. Our results showing a higher percentage of $\mathrm{CD}_{3}+$ / CD200+ cells in GC with a significantly lower CD3+CD200R+ cell percentage could indicate the general suppression of CD3+-related immunity as a result of CD200/CD200R action on T lymphocytes. Wang and Shen found that the percentage of CD8+ cells was higher among lymphocytes in advanced gastric cancer compared to early tumors, but the percentage of CD4+ cells appeared to be lower compared to the respective groups [15]. We demonstrated that in advanced GC, there is a higher rate of CD4+CD200+ cells as well as CD8+CD200+ cells with a lower percentage of CD4+CD200R+ and CD8+CD200R+ cells compared to healthy controls. This could indicate that CD8+ cytotoxic cells could be functionally impaired by CD200/CD200R inhibition in advanced GC. Similar results were observed in programmed death-1 (PD-1) expression on CD4+ and CD8+ T cells from GC patients, which was significantly higher than that from normal controls. It was also related to disease progression. PD-1 expression on CD4+ and CD8+ T cells from gastric cancer tissue was significantly higher than that from normal gastric mucosa and peripheral blood. Saito et al. also reported that PD-1 positive CD4+ and CD8+ T cells produced significantly less IFN- $\gamma$ than PD-1 negative $T$ cells [16]. Our results indicate a possible similar mechanism of immunosuppression by the CD200+/CD200R+ axis. The higher percentage of CD4+CD200+, which positively correlated with the percentage of CD8+CD200+ cells, may be, in part, responsible for the immune evasion in advanced gastric cancer.

Strongly significant differences were observed between CD19+/CD200+ cells in the GC group and healthy control group. Almost the entire subset of peripheral CD19+ cells was positive for CD200+ expression in the GC group with the coexistence of a significantly lower percentage of CD19+/ CD200R+ cells. This finding could indicate the importance of CD200 molecule up-regulation in immune function of $B$ lymphocytes in gastric cancer. No previously published studies concerning the frequency of CD200(+)-expressing B-lymphocytes in solid tumors are available. The high percentage of CD19+CD200+ cells in peripheral blood in GC patients corresponds to previous data from non-solid tumor research, that is, B-cell chronic lymphocytic leukemia (B-CCL). CD200 showed high expression on a CD5/19-positive clone in all B-CLL patients, with a mean of $94 \%$. CD200 was also brightly expressed on $96 \%$ and $99 \%$ of CD19+ cells in cases of hairy cell leukemia [17]. Further studies are needed to compare these results with other digestive tract solid tumors, i.e. colon adenocarcinoma, to confirm high frequency of CD19+CD200+ cells in peripheral blood. As shown in previous studies, the percentage of CD19+ cells in peripheral blood from healthy controls and gastric cancer patients is similar. However, a significantly increased percentage of CD19+CD24hiCD38hi Bregs was observed in gastric cancer patients. Increased CD19+CD24hiCD38hi Bregs positively correlate with the levels of CD4+FoxP3+ Tregs and play a significant immunosuppressive role in gastric cancer. Both Bregs and total B cells could promote cancer growth mainly by inhibiting the cytotoxic activity of Th1/CD8+ cells, which was mediated by IL-10 and TGF- $\beta$ production $[18,19]$. Our results presenting predominance of CD19+CD200+ cells with a lower percentage of CD19+CD200R+ cells in the GC group compared to the healthy control group may suggest the involvement of CD200/ CD200R signaling in immunosuppressive action of B lymphocytes in GC. We also observed higher expression of CD200 and CD200R antigens on CD19+ cells in GC patients compared to controls with a positive correlation of CD200 and CD200R MFI values in GC patients. These findings also support the possible involvement of these molecules in deterioration of B lymphocyte-mediated humoral immunity in advanced GC.

Our results demonstrate the status of the CD200 molecule on PB lymphocytes in patients with advanced GC. The studied group of patients was rather homogeneous with the prevalence of UICC stage III/IV. However, patients were enrolled in the analysis consecutively, representing the regular, most commonly treated group in the Polish/ Central-East Europe population, primarily diagnosed with gastric cancer [20]. Our preliminary results showed no difference in the percentage of CD200+ lymphocytes in patients staged UICC II vs. III/IV. It may suggest that a possible mechanism of immune evasion in GC, which could involve CD200/CD200R signaling, appears soon in tumorigenesis. Research on CD200 status on lymphocytes in patients with early gastric cancer would demonstrate a wider perspective on the possible immune deregulation in the course of this devastating disease. Higher expression of the CD200R molecule on CD8+ peripheral cells in diffuse-type compared to intestinal-type cancer patients indicates possible involvement of CD200-receptor mediating action in more aggressive tumorigenesis, with a poor prognosis. 
Hence it would also be beneficial to study the status of CD200/CD200R expression and its potential role on tumor cells and tumor-infiltrating lymphocytes, especially on CD8+ T cells, which are able to directly kill cancer cells. Several previous reports have documented a strong association between tumor-infiltrating CD8+ T cells and patient survival in many types of cancers [21]. Rygiel et al. indicate that CD200/CD200R signaling suppresses anti-tumor responses independently of CD200 expression on the tumor. In an experimental model, CD200-negative mice were resistant to chemical skin carcinogenesis. Moreover, CD200R controlled tumor growth independently of CD200 expression by the tumor cells themselves. CD200(-) mice did not acquire tolerance to antigens, suggesting that tumor rejection was suppressed through CD200-induced immune tolerance. Higher expression of proinflammatory cytokines including IL- $1 \beta$ and IL- 6 by dendritic cells was observed together with reduced tumor growth [22]. Recently published results also point to a critical role for CD200R signaling in limiting the growth and metastasis of CD200+ tumors. Liu et al. found that CD200R-deficient mice exhibited fast growth of CD200+ but not CD200(-) melanoma tumors. CD200R-deficient mice receiving CD200(+) tumor cells showed massive primary tumor growth and multiple remote metastases, whereas the growth of the same tumors in wild-type mice was limited. CD200(+) tumors in CD200R-deficient mice contained higher numbers of CD11b(+)Ly6C(+) myeloid cells, exhibited increased angiogenesis, and showed reduced infiltration of CD4+ and CD8+ T cells. The authors assume that targeting CD200R signaling may potentially interfere with the metastatic growth of CD200+ tumors [23]. These results support the importance of future studies on CD200 blockade in the treatment of neoplastic tumors. At present, several monoclonal antibodies (mAbs) are applied to treat patients with solid tumors. Because the CD200 molecule does not have a signaling domain, it can be assumed that anti-CD200 mAbs do not directly affect targeted tumor cells. It was shown that anti-CD200 mAbs did not attenuate tumor cell growth or directly induce cell death. However, anti-CD200 Abs efficiently mediate Ab-dependent cellular cytotoxicity of activated T cells, critical cells involved in immune-mediated killing $[24,25]$. Neutralization of expressed CD200 by anti-CD200mAbs decreased the tumor metastasis in the EMT6 mouse breast cancer line [26].

In conclusion, we demonstrated for the first time that in advanced gastric cancer, the CD200 molecule is expressed on a higher percentage of CD3+, CD4+, and CD8+ cells in peripheral blood than in the healthy control group, and it was accompanied by a lower percentage of CD200R ex- pressing cells in the GC group. We also found that almost the entire subset of CD19+ B cells presented CD200 expression in the GC group with a significantly lower percentage of CD19+CD200R+ cells in GC patients vs. healthy controls. Higher frequency of ligand (CD200)-expressing cells and lower frequency of receptor (CD200R)-expressing cells resulting from interaction between cancer cells and the immune system of the host indicate possible impairment of regulatory function of lymphocytes, leading to chronic inflammation and carcinogenesis. These results confirm the importance of CD200/CD200R signaling in attenuation of the immune response in GC. Further studies are needed to clarify the presence of CD200+/ CD200R+ lymphocytes in the tumor microenvironment, as well as the CD200 molecule on the surface of gastric cancer cells.

\section{Conflict of interest}

The authors declare no conflict of interest.

\section{References}

1. Jemal A, Bray F, Center MM, Ferlay J, Ward E, Forman D. Global cancer statistics. CA Cancer J Clin 2011; 61: 69-90.

2. Zou W. Regulatory T cells, tumour immunity and immunotherapy. Nat Rev Immunol 2006; 6: 295-307.

3. Anandkumar A, Devaraj H. Tumour immunomodulation: mucins in resistance to initiation and maturation of immune response against tumours. Scand J Immunol 2013; 78: 1-7.

4. Gajewski TF, Meng Y, Blank C, et al. Immune resistance orchestrated by the tumor microenvironment. Immunol Rev 2006; 213: 131-45.

5. Grogg KL, Lohse CM, Pankratz VS, Halling KC, Smyrk TC. Lymphocyte-rich gastric cancer: associations with Epstein-Barr virus, microsatellite instability, histology, and survival. Mod Pathol 2003; 16: 641-51.

6. Dunn GP, Old LJ, Schreiber RD. The three Es of cancer immunoediting. Annu Rev Immunol 2004; 22: 329-60.

7. Barclay AN, Clark MJ, McCaughan GW. Neuronal/lymphoid membrane glycoprotein MRC OX-2 is a member of the immunoglobulin superfamily with a light-chainlike structure. Biochem Soc Symp 1986; 51: 149-57.

8. Wright GJ, Jones M, Puklavec MJ, Brown MH, Barclay AN. The unusual distribution of the neuronal/lymphoid cell surface CD200 (OX2) glycoprotein is conserved in humans. Immunology 2001; 102: 173-9.

9. Wong KK, Khatri I, Shaha S, Spaner DE, Gorczynski RM. The role of CD200 in immunity to B cell lymphoma. J Leukoc Biol 2010; 88: 361-72.

10. Moreaux J, Hose D, Reme T, et al. CD200 is a new prognostic factor in multiple myeloma. Blood 2006; 108: 4194-7.

11. Petermann KB, Rozenberg GI, Zedek D, et al. CD200 is induced by ERK and is a potential therapeutic target in melanoma. J Clin Invest 2007; 117: 3922-9.

12. Stumpfova M, Ratner D, Desciak EB, Eliezri YD, Owens DM. The immunosuppressive surface ligand CD200 augments the metastatic capacity of squamous cell carcinoma. Cancer Res 2010; 70: 2962-72. 
13. Sobin LH, Gospodarowicz MK, Wittekind C. TNM classification of malignant tumours. $7^{\text {th }}$ ed. West Sussex (UK). Wiley-Blackwell 2009.

14. Lee AJ, Kim SG, Chae HD, Lee GH, Shin IH. gammadelta $\mathrm{T}$ cells are increased in the peripheral blood of patients with gastric cancer. Clin Chim Acta 2012; 413: 1495-9.

15. Wang L, Shen Y. Imbalance of circulating T-lymphocyte subpopulation in gastric cancer patients correlated with performance status. Clin Lab 2013; 59: 429-33.

16. Saito H, Kuroda H, Matsunaga T, Osaki T, Ikeguchi $M$. Increased PD-1 expression on CD4+ and CD8+ T cells is involved in immune evasion in gastric cancer. J Surg Oncol 2013; 107: 517-22.

17. El Desoukey NA, Afify RA, Amin DG, Mohammed RF. CD200 expression in B-cell chronic lymphoproliferative disorders. J Investig Med 2012; 60: 56-61.

18. Wang WW, Yuan XL, Chen H, et al. CD19+CD24hi CD38hiBregs involved in downregulate helper $T$ cells and upregulate regulatory $T$ cells in gastric cancer. Oncotarget 2015; 6: 33486-99.

19. He Y, Qian H, Liu Y, Duan L, Li Y, Shi G. The roles of regulatory B cells in cancer. J Immunol Res 2014; 2014: 215471.

20. Gurzu S, Orlowska J, Sugimura H, et al. Immunohistochemical features and staging of early gastric cancer. Arch Med Sci 2017; 13: 1373-82.

21. Pages F, Galon J, Dieu-Nosjean MC, Tartour E, SautesFridman C, Fridman WH. Immune infiltration in human tumors: a prognostic factor that should not be ignored. Oncogene 2010; 29: 1093-102.

22. Rygiel TP, Karnam G, Goverse G, et al. CD200-CD200R signaling suppresses anti-tumor responses independently of CD200 expression on the tumor. Oncogene 2012; 31: 2979-88.

23. Liu JQ, Talebian F, Wu L, et al. A critical role for CD200R signaling in limiting the growth and metastasis of CD200+ melanoma. J Immunol 2016; 197: 1489-97.

24. Kretz-Rommel A, Qin F, Dakappagari N, et al. CD200 expression on tumor cells suppresses antitumor immunity: new approaches to cancer immunotherapy. J Immunol 2007; 178: 5595-605.

25. Kretz-Rommel A, Qin F, Dakappagari N, Cofiell R, Faas SJ, Bowdish KS. Blockade of CD200 in the presence or absence of antibody effector function: implications for anti-CD200 therapy. J Immunol 2008; 180: 699-705.

26. Gorczynski RM, Clark DA, Erin N, Khatri I. Role of CD200 expression in regulation of metastasis of EMT6 tumor cells in mice. Breast Cancer Res Treat 2011; 130: 49-60. 